\title{
Native populations and the opioid crisis: forging a path to recovery
}

\author{
Martina Whelshula ${ }^{1}$ - Margo Hill ${ }^{2}$ - S. E. Galaitsi ${ }^{3}$ (D) Benjamin Trump ${ }^{3} \cdot$ Emerson Mahoney $^{3} \cdot$ Avi Mersky $^{3,4}$. \\ Kelsey Poinsatte-Jones ${ }^{3,5} \cdot$ Igor Linkov $^{3}$
}

Accepted: 20 March 2021 / Published online: 21 April 2021

(c) The Author(s), under exclusive licence to Springer Science+Business Media, LLC, part of Springer Nature 2021

\begin{abstract}
American Indian/Alaska Native (AI/AN) populations have proven particularly susceptible to the opioid crisis in the USA, but the White House's 2019 national opioid policy roadmap is not structured to address AI/AN vulnerabilities. The concept of resilience, usually considered a positive system attribute, can be applied to complex systems to understand the larger compensatory interactions that restore systems to previous structures despite disruptions or interventions. The opioid crisis is a case of detrimental resilience because even effective interventions have not succeeded in eradicating opioid abuses. Resilience-based systemic interventions are needed to disrupt various aspects of systems while enhancing the social and cognitive abilities of affected populations to withstand the threat. This paper examines community characteristics, healthcare, and law enforcement within the context of AI/AN populations to emphasize the mechanisms that promote undesirable resilience for the opioid crisis. A research agenda bringing together systems science and management is needed to coordinate sectoral interventions and establish strategies to disrupt the resilient cycle of opioid addiction.
\end{abstract}

Keywords Opioids $\cdot$ American Indians $\cdot$ Systems management $\cdot$ Resilience

\section{Introduction}

Efforts to mitigate the opioid crisis in the USA must include immediate research and policy attention to its disproportional impact on Native populations. In 2015, American Indians/Alaska Natives (AI/AN) had the highest drug overdose death rates of any population in the USA (Mack et al. 2017). The opioid mortality rates for AI/AN populations have risen almost continuously for nearly two decades and are comparable to the mortality rates of non-Hispanic whites (Tipps et al. 2018). Figure 1 illustrates overdose deaths, including opioids, over time by race.

S. E. Galaitsi

Stephanie.Galaitsi@usace.army.mil

1 NARCH X, Spokane, WA 99202, USA

2 Eastern Washington University, Spokane EWU Center 384, Spokane, WA 99202, USA

3 US Army Corps of Engineers, 696 Virginia Rd, Concord, MA 01704, USA

4 Now Working At One Lomb Memorial Drive, Rochester, NY 14623, USA

5 Kwant.ai 335 Madison Ave, New York, NY 10017, USA
Furthermore, AI/AN drug overdose rates may be underestimated by as much as 35\% because of race/ethnicity misclassifications on death certificates (Mack et al. 2017). Such acute manifestations of the opioid crisis for AI/AN populations make its related research management an urgent concern. Even though the White House Office of Science and Technology Policy's October 2019 national roadmap to stem the opioid crisis (Fast Track Action Committee on Health Science and Technology Response to the Opioid Crisis, Committee on Science of the National Science \& Technology Council 2019) recognizes that the special needs of specific groups should be studied, it does not sufficiently consider ways to prevent the problem's systemic roots from propagating through interconnected sectors.

The complexities contributing to the opioid crisis require systems science to address the root causes of opioid use. Resilience, defined as ability of a system to recover from disruptions, is usually considered a positive system attribute. However, the opioid crisis is an example of detrimental resilience, as shown by the multiple interventions that have not succeeded in ending opioid abuses. Below we describe some successful interventions, couched by the framing we provide in this paper, but the persistence of opioid abuse among AI/AN communities can be attributed to 


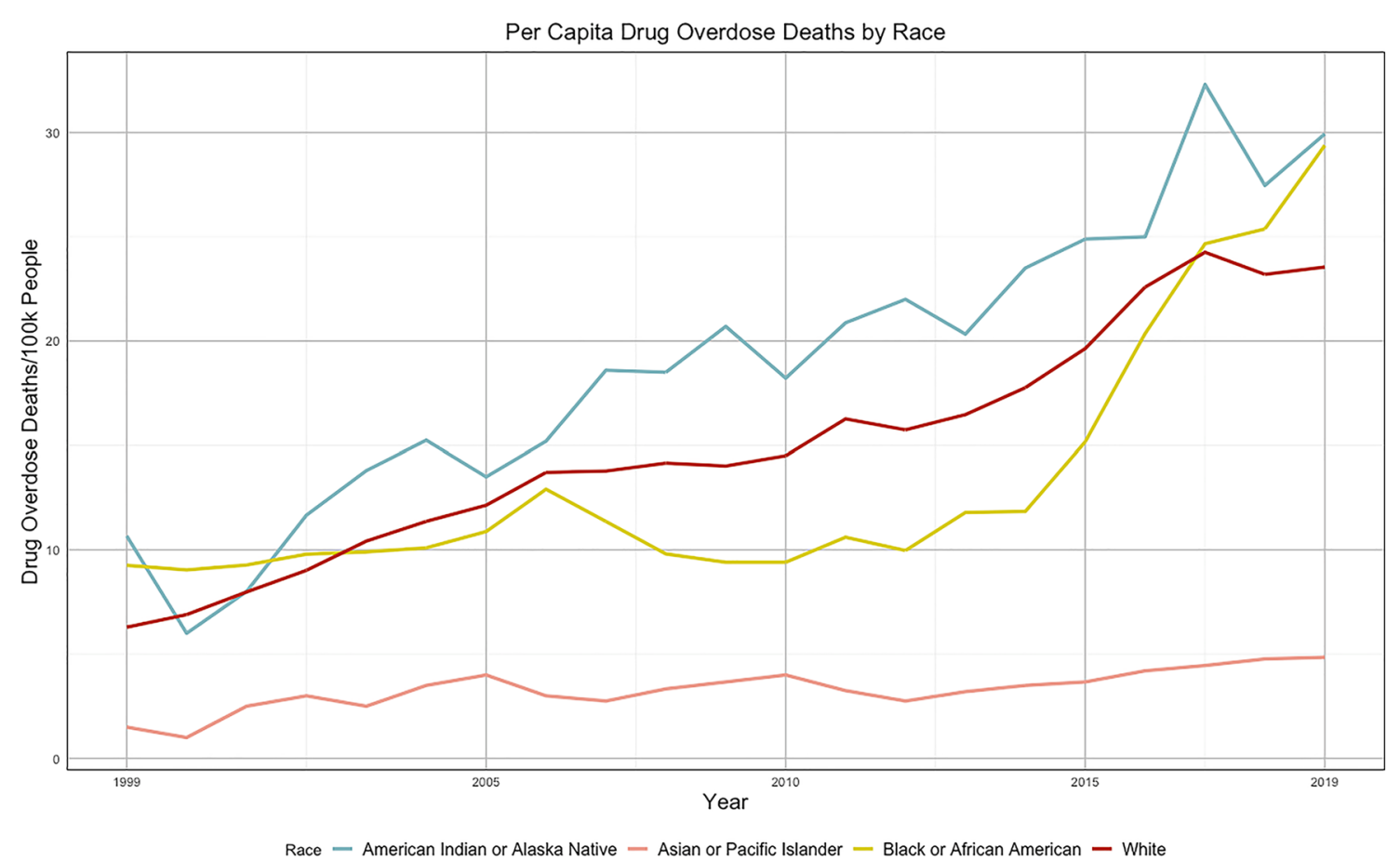

Fig. 1 Overdose deaths over time by race (CDC Wonder Online Database 2020)

the undesired resilience of the opioid abuse system. Thus, resilience science can provide an analytical perspective to reveal strategies for reducing the resilience of opioid supply chains, consumer demand, and subsequent high mortality rates. Resilience-targeted interventions are needed to disrupt various aspects of systems while enhancing the social and cognitive abilities of affected populations to withstand the threat.

We argue that the opioid crisis and its resilience in AI/ AN populations arises from the relationships between federal Indian law, policies, and societal practices. Herein we examine three sectors affected by these differences: community, healthcare, and law enforcement. We discuss the unique characteristics of these sectors for AI/AN populations, and how these differences influence opioid resilience. By applying a network lens, we can better understand the challenges related to opioid use and propose strategies specific to AI/AN communities for forging pathways towards both recovery and abstention from opioid abuse for vulnerable populations.

\section{Sectors of the opioid crisis}

\subsection{Community}

Park et al. (2020) outlines community as an underutilized area in which the crisis can be solved. The community sector encompasses the conditions in which people live, commute, and work. Factors exposing community members to risks of opioid addiction may include physical activities with risks, like driving, playing sports, or performing manual labor, where accidents can necessitate prescription pain medication. But beyond voluntary physical risks, the National Academy of Sciences and Medicine (2017) considers the root causes of opioid misuse to be poor working conditions, lack of economic opportunity, and eroded social capital. We examine these factors in this section. They vary between communities and often along lines of race/ethnicity, including AI/AN. There are 574 federally recognized tribes in the USA that are considered sovereign nations (Robbins 2020), and these factors vary between them as well, but have some commonalities, especially as related to federal policies of $\mathrm{AI} / \mathrm{AN}$ populations. With regards to working conditions in AI/AN communities, the Occupational Safety and Health Administration (OSHA) generally applies to tribal enterprises (Occupational Safety and Health Administration (OSHA) 1998). Economically, however, depressed economic sectors are common in reservations, leaving residents with insufficient opportunities; Brown et al. (2017) argue that uncertainty in law enforcement, as will be described below, negatively impacts economic development in tribal communities. Casinos, entrepreneurial endeavors, arts, tourism, and external investment providing employment for reservations provide a few examples of exceptions, with regards to depressed economic sectors, on certain reservations (Emery 
et al. 2006). Illustrating, the extreme level of inter-ethnicity income inequality, the poverty rates in 2019 for white people and AI/AN people were $9.0 \%$ and $24.2 \%$, respectively (KFF 2020). Furthermore, between 2006 and 2010, about $45 \%$ of working age Native Americans were employed, while the average was nearly $60 \%$ for Americans as a whole (Kocherlakota 2017).

The history of social capital erosion in tribal reservations likely represents the largest departure for AI/AN populations from other US communities in terms of the National Academy's three roots of opioid addiction. AI/AN social capital was specifically targeted in policies for Native people in the nineteenth and twentieth centuries. Until the passage of the Indian Child Welfare Act in 1978, federal programs systematically removed Tribal children from their families to attend federally run boarding schools. These schools aimed to assimilate $\mathrm{AI} / \mathrm{AN}$ children into mainstream American culture at the expense of their culture, languages, proximity to family, and often their own safety. Such assimilations sought to disrupt the native cultures that persisted on reservations. This and other social traumas have had intergenerational effects (Lajimodiere 2014), visible in reservations' insufficient community support structures and violence or drug use within homes (Stevens et al. 2015), where substance use by family members constitutes a risk factor for other members of the family (Substance Abuse and Mental Health Services Administration 2018). The community impacts continue to be seen: AI/AN children born to parents using opioids struggle more to develop and thrive, and a parent's negligence, arrest, or death may instigate their children's removal from their homes and in some cases from their communities for foster care (Momper et al. 2012). Compounding this, studies of Canadian indigenous mothers found that those who had a child removed were 55\% more likely to have overdosed after adjusting for education and indigenous ancestry (Thumath et al. 2020). Any strategy seeking to holistically protect AI/ AN populations from the opioid crisis will have to reckon with the intergeneration trauma and the legacy of pain that affect social capital and leave AI/AN populations more vulnerable to the factors leading to substance disorders. Opioid abuse for $\mathrm{AI} / \mathrm{AN}$ populations is resilient because the demand is bolstered by these circumstances.

Scholars and practitioners recognize the need for community-based approaches to address the opioid crisis. These could include drug checking programs and appointing PWUD (persons who use drugs) to leadership positions for designing anti-opioid strategy and policy may help (Park et al. 2020). Leston et al. (2019) suggest implementing prevention and help services into school curriculum. Childs et al. (2021) indicate that local champions and leaders who advocate for drug harm reduction strategies that provide overdose education and safer alternative drugs may reduce stigma against PWUD. Conversely, resistance to harm reduction strategies, a particularly salient issue in rural communities, can be a major impediment to both eliminating stigma against PWUD and opioids overdoses generally (Childs et al. 2021).

\subsection{Healthcare}

Our motivation to include healthcare as an important avenue for countering opioids abuse stems from issues of normalization of drug use as a medical issue, medical training (Park et al. 2020), pharmaceutical company emotional support deficiencies (Leston et al. 2019), and sentiment surrounding minority treatment in healthcare (Goodman et al. 2017).

The over-prescription of opioids are a fundamental cause of the epidemic: from 2000 to 2010, opioid prescription for oral analgesics rose 104\% (Sarpatwari et al. 2017). After pharmaceutical companies marketed time-released opioids like OxyContin as having low risk of addiction (Gounder 2013; Meldrum 2016), health professionals around the USA, including dentists (see Larson 2019) and doctors working for the Indian Health Service (IHS) prescribed them more readily. But opioids proved highly addictive, and the increased prescriptions lead to widespread opioid misuse (U.S. Health and Human Services 2019).

In rural communities, including native communities, opioid addiction problems can arise from healthcare deficiencies. Opioids are also more likely to be prescribed in counties with more uninsured people (Center for Disease Control (CDC) 2019), and even people that have insurance may find that prescription narcotics are more reliably covered than other medical tools (Gounder 2013), such as corrective surgery, which are often considered too costly for economically depressed and low density populations (Meldrum 2016). Compounding these factors, Indian Health Clinics are severely underfunded: in 2017, national health expenditures per capita were $\$ 9726$ compared with $\$ 4078$ for IHS patients (IHS.gov 2019). Clinics are often placed on Priority One status to provide coverage only when life and limb are at immediate risk. Sick or injured patients who are not covered for treatment of the cause of pain instead receive options to manage it, often opioids. Significant healthcare reform, described below, has reduced the quantity of opiates distributed to individual patients, but these regulations alone will not eliminate the need to address medical symptoms and causes for over 25 million Americans experiencing daily pain. Opioid availability is resilient because few alternatives exist for pain management. When opioids are not made available through the health sector, users can obtain access through illegal drug markets.

Interventions in the medical field include training medical professionals to use opioids appropriately and preventing pharmaceutical companies from paying doctors to advertise these drugs in the media and to patients (Park et al. 2020). 
Leston et al (2019) suggests expanding behavioral health services to address emotional issues, such as those discussed in the previous section, which have been reported as a major cause for PWUD.

\subsection{Law enforcement}

The opioid literature emphasizes the need for anti-policing progress in the fight to address the opioids abuse. Minhee and Calandrillo (2019) attribute the criminal-based stigma to the Reagan-era drug policy and the war on drugs, and contrast the USA with countries like Switzerland and Portugal, where crimes, addiction rates, and deaths decreased following the elimination of expensive and inefficient criminal charges as a solution to drug problems. Conversely, in the USA, the criminal justice system's attempts at interdiction of illicit opioids have fueled the emergence of potent and less bulky drug products (Dasgupta et al. 2018) more likely to escape detection. This has enabled resilience in illicit markets operations. Incarcerating offenders does not necessarily protect them because they emerge with a subsequent higher risk of overdosing, partially due to decreased tolerance (Joudrey et al. 2019). Additionally, having a public record from a drug conviction hinders employment opportunities that could help draw users away from opioid use (Dasgupta et al. 2018), again making the illicit market more resilient. Poor economic prospects can also encourage illegal dealing of opioids: for example, in a small-sample study of a Great Lakes Indian Reservation, Momper et al. (2012) found that elder and middle-aged community members with legitimate proscriptions sold pills to augment their incomes.

In a system where legal recourse is already mismatched to the problem, an additional layer of complexity exists in Indian Country through the strict dictates of the various authorities that can enforce laws on tribal members and non-members. For major crimes, the federal government is responsible for investigation and prosecution, but in recent years the U.S. Attorney's Office has declined to prosecute a relative steady rate of one third of the cases referred from Indian Country (U.S. Department of Justice 2017). Criminal justice officials within local communities lack the resources and support to surveil as necessary to prevent movements and impacts of drugs. Furthermore, complicated jurisdictional schemes make it difficult to penalize drug dealers, especially if they come from outside a reservation: tribes do not have criminal jurisdiction over non-Indians (1978). Thus, illegal behavior can persist in tribal areas due to jurisdictional constraints (Crane-Murdoch 2013) and well as financial and manpower constraints within the community police. The system of opioid demand can perpetuate itself while benefitting from legal impunity.

Park et al. (2020) identify the reversal of the criminalization of PWUD in their six-part framework for addressing the opioids epidemic. Often times, witnesses to overdoses state fear of criminal action as a reason for the delay or refusal to call emergency services after an overdose. In fact, geographic areas with higher rates of police activity have higher rates of overdose fatalities (Park et al. 2020). Good Samaritan laws could help eliminate reporting fear (Leston et al. 2019). Lastly, the prosecutorial mindset towards drug use impedes harm reduction strategies (Childs et al. 2021) that could help PWUD.

\section{Interventions for ai/an populations}

In describing these sectors, we demonstrate how federal Indian law, policies, and societal practices contribute to the opioid crisis and its resilience in AI/AN populations. This contrasts to attributing drug use to any physiological differences of $\mathrm{AI} / \mathrm{AN}$ populations, a narrative that has proven harmful to efforts to curb alcohol use (Gonzalez et al. 2019). Understanding how the sectors and their interactions enable resilience in opioid availability and use provides inside for opportunities to disrupt that resilience.

Figure 2 below shows the interplay between the three sectors supporting resilience in opioid use. Opioids serve as a vector that converts social problems to costs for local communities, health care providers, and law enforcement. In the system of opioid resilience, each sector feeds off systemic problems in other sectors.

The healthcare and law enforcement sectors have each taken steps to combat the US opioid crisis. The national prescription rate of opioids has decreased from its peak in 2012 ( 81.3 per 100 persons in the USA), and law enforcement seizes drugs, arrests offenders, and administers naloxone to prevent overdose deaths. Yet the rate of deaths from opioids has continued to rise (Scholl et al. 2019). While the root causes of the crisis remain unaddressed, the system of opioid abuse remains resilient. AI/AN communities are uniquely vulnerable and afflicted in the crisis and thus offer insightful perspectives of potential actions that might be taken to reduce the resilience of opioid usage and support community recovery.

Current interventions for AI/AN populations show an understanding of the multi-dimensionality of the opioid crisis and the interactions of the three sectors profiled in this paper. Tipps et al. (2018) document different practices of various $\mathrm{AI} / \mathrm{AN}$ communities in their work to combat the opioid crisis, and tribes have been leading community-based responses that incorporate public health data infrastructure (Seven Directions: A Center for Indigenous Public Health 2019). In the health sector, IHS is the first federal agency to centralize information about drug prescriptions to curtail doctor shopping practices. Changes in local IHS administration can give more autonomy to tribes, though this may 


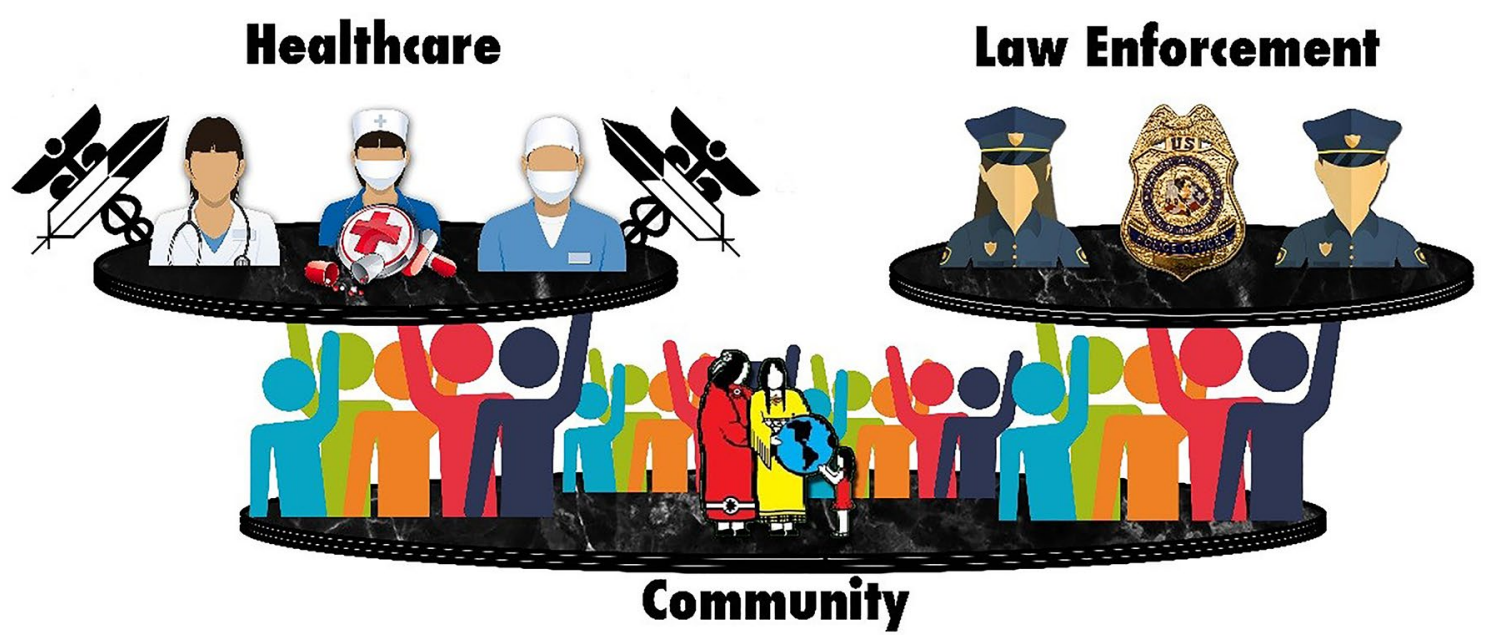

Fig. 2 Sectors of the opioid crisis

not always be feasible because of resource constraints. But doing so allows for treatment options that include grounding in cultural traditions and building social capital to address opioid resilience. Since the onset of the SARS-CoV-2, telemedicine expansions in Canada and the USA has increased access to opioid treatment for indigenous communities, decreased stigma, and promoted patient self-efficacy (Wendt et al. 2021). Yet another example of increased autonomy of tribes in health care is exemplified by the Native Behavioral Health Access Improvement Act, designed to allow tribes to incorporate cultural and behavioral practices into their recovery programs (Smith 2019).

For law enforcement, many tribes have enacted Wellness Courts, which transfer drug-offending tribal members from criminal proceedings to culturally appropriate clean and sober treatment programs. These efforts represent solutions that address two sectors within the opioid crisis, law enforcement and community. Tribes can also banish individuals from reservations, one of the few legal recourses available to tribes over non-tribe members, as a way to sever connections with drug traffickers (Tipps et al. 2018). Unfortunately, drugrelated banishment has proved difficult to enforce because of inadequate policing resources, so the method does not necessarily benefit the community. Furthermore, Well Courts have demonstrated some limitations. Notably, the most successful participants in the courts are typically those that leave their reservations after the program is finished, which is counterproductive in preserving and maintaining native community, culture, and values (Cochran and Kettel 2020). Additionally, the success of these court programs varies widely between tribes, and state court programs have higher rates of success (Cochran and Kettel 2020).

Tribes have also sued drug companies, which could provide resources for treatment options, but the legality of such cases have been contentious in the courts and even a victory would be unlikely to halt the ongoing crisis (Tipps et al. 2018). The effectiveness of any of these actions can be marred by insufficient resources, but tribes throughout the country have begun implementing programs to support their members in avoiding or controlling opioid addiction. The Cherokee Nation implemented their own comprehensive health care system in Oklahoma, taking extreme precaution to limit the supply of opioids to tribal people. Despite this, they continued to see higher rates of opioid overdoses, which their data collection system was able to attribute to external sources of opioids supply. They have proceeded to file a lawsuit against pharmaceutical companies for contributing to their increase in addiction and death rates (Leeds 2018).

In the community sector, a key systemic strategy must include early intervention with pre-school to high school children impacted by intergenerational trauma or intergenerational post-traumatic stress disorder (PTSD). Children with PTSD can experience a variety of anxiety disorders and can enter adolescence with unhealthy coping responses. Experimentation with alcohol and drugs can relieve anxieties and lead to addiction. Key systems within tribal communities for training can include tribal Head Start programs, childcare and public or tribal schools. Anxiety disorders in Native American children are largely unknown and, therefore, require training and skill development for teachers and parents. Training would assist teachers and parents in identifying key behavior associated with anxiety and provide skills in managing the symptoms. These trainings would elevate the collective knowledge and skill level of a community to address key mental health problems and, thereby, reduce the resilience of opioid use.

MAT (medication-assisted treatment) has been a growing method to reduce addiction and overdose in both tribal populations and in general in the USA. Vashishtha et al. (2017) suggest that federal and state level funding must be 
increased to address $92 \%$ of the opioid-dependent population eligible for MAT treatment. They also address the need to remove barriers to MAT prescription and the prioritization of low -threshold and experimental MAT approaches for marginalized populations. The implementation of MAT programs does not come without challenges, though. MAT programs are often met with resistance because they are not seen as a form of sobriety. Framing of MAT as a treatment for addiction like a biological disease and recognizing it as a legitimate form of medical treatment is needed to help communities accept MAT services. Venner et al. (2018) also discussed shortcomings of MAT strategies that include inconsistency with medicine-free traditional AI/AN methods of healing, barriers to MAT access in the form of discrimination and high turnover within IHS, and a lack of research on MAT.

Tribes have implemented specific programming according to their needs, strengths, and resources. The Penobscot Nation Wellness Court engages participants in their culture and community. In "open court nights," members of the greater community share awareness and broader perspectives surrounding addiction for topics like historic trauma or brain functionality that are relevant to participants' experiences (Decontie 2018). The Lummi tribe police seek to provide positive role models and even mentorship for the community. Health providers and law enforcement are able to coordinate, which can help more individuals to enroll in the available treatment programs (Long 2018).

The Suquamish has implemented several programs and initiatives to encourage and support recovery, including intentional prevention like youth cultural programming, trips, and education opportunities that not only create an atmosphere of accountability but also trust and relationships between individuals and their community. There are also programs to provide life skills for adults and behavioral health, including for stress and unresolved grief. The goal is to invest in living a clean and healthy life based on the tribe's culture, traditions and spirituality (Forsman 2018). The Warm Springs Tribal Council has a community counseling center and a family preservation program that seeks to tap into tribal traditions and spirituality. Funding was recently allocated to develop curriculum on historic trauma for school-aged children and families to learn to talk about uncomfortable but important topics, including as a means of drug use prevention. The efforts to reach people include visits to jails, and speaking with the youth as well as the elderly (Miller 2018). In a community-based needs assessment, successes included scenarios where treatment involved clients reconnecting to the community and culture, communication with clinicians, case managers, and patients was present, and cases where known barriers to successful treatment were faced head on, like stigma, privacy, distance, insurance, and readiness to change (Zeledon et al. 2020).
These interventions could be strengthened by a resilience analysis of the opioid system that maps the various pathways between demand and supply to anticipate how they will shift in response to a disruption. The problem cannot be solved in siloes; no single policy solution can disrupt the resilience of the opioid crisis, whether as a legal issuance of prescribed opioids or by targeting the black market. An effective policy or practice to reduce the resilience of the opioid supply chain requires first that causal factors causing resilience be better understood. Following this, a resiliency framework to analyze cross-system intervention strategies can target both the causes and ongoing prevalence of opioid addiction.

\section{Conclusions}

Opioid abuse affects the fabric of entire communities and its resilience arises from interacting factors between different sectors. While risk-informed management strategies focus on identifying the most vulnerable component of a system to disrupt, such one-dimensional efforts within opioid supply and demand networks have proven insufficient because these systems can restructure and compensate to retain functionality. Solutions must transcend individual users to examine the interactions and reinforcing nature of different societal sectors. Although government documents continue to view the justice system as distinct from other non-biological contributors of opioid addiction (Fast Track Action Committee on Health Science and Technology Response to the Opioid Crisis, Committee on Science of the National Science \& Technology Council 2019), opioid use must be addressed through holistic systems management.

The opioid distribution and demand system will benefit from more intentional analysis. The crisis has a broad foundation, and requires making resources available, like inpatient treatment centers or other clean and sober housing, or further support financial for existing programs. Cohesive policy guidance to address the opioid crisis must recognize the multi-dimensionality of the crisis and deploy systematic anti-resilience strategies.

Disclaimer The views, thoughts, and opinions presented in this article belong solely to the authors, and not necessarily to the author's employer, organization, committee or other group or individual.

\section{References}

Brown JR, Cookson JA, Heimer RZ (2017) Courting economic development. World Bank Econ Rev 30:S176-S187

CDC Wonder Online Database (2020) Multiple Cause of Death Files, 1999-2019, as compiled from data provided by the 57 vital statistics jurisdictions through the Vital Statistics Cooperative Program. 
Center for Disease Control (CDC) (2019) Opioid overdose: data: prescribing practices. Center for Disease Control, Atlanta

Childs E, Biello KB, Valente PK et al (2021) Implementing harm reduction in non-urban communities affected by opioids and polysubstance use: a qualitative study exploring challenges and mitigating strategies. Int J Drug Policy 90:103080

Cochran MM, Kettel CL (2020) Rehabilitative justice: the effectiveness of healing to wellness, opioid intervention, and drug courts. Am Indian Law J 9:4

Crane-Murdoch S (2013) On Indian land, criminals can get away with almost anything. The Atlantic 22:

Decontie R (2018) Court Clerk and Cultural Advisor of Penobscot Nation

Dasgupta N, Beletsky L, Ciccarone D (2018) Opioid crisis: no easy fix to its social and economic determinants. Am J Public Health 108:182-186

Emery M, Wall M, Bregendahl C, Flora C (2006) Economic development in Indian country: redefining success. Online J Rural Res Policy 1:1

Fast Track Action Committee on Health Science and Technology Response to the Opioid Crisis, Committee on Science of the National Science \& Technology Council (2019) Health research and development to stem the opioid crisis: a national roadmap

Forsman L (2018) Chairman of the suquamish tribe.

GonzalezBravo VMAJ, Crouch MC, Team PSS (2019) Endorsement of the "firewater myth" affects the use of protective behavioral strategies among American Indian and Alaska Native students. Addict Behav 93:78-85

Goodman A, Fleming K, Markwick N et al (2017) "They treated me like crap and I know it was because I was Native": the healthcare experiences of aboriginal peoples living in Vancouver's inner city. Soc Sci Med 178:87-94

Gounder C (2013) Who is responsible for the pain-pill epidemic. New Yorker 8:

IHS.gov (2019) IHS Profile Factsheet

Joudrey PJ, Khan MR, Wang EA et al (2019) A conceptual model for understanding post-release opioid-related overdose risk. Addict Sci Clin Pract. https://doi.org/10.1186/s13722-019-0145-5

KFF (2020) Poverty Rate by Race/Ethnicity

Kocherlakota N (2017) What's different about economic development in Indian country? Federal Reserve Bank of Minneapolis

Lajimodiere DK (2014) American Indian boarding schools in the United States: a brief history and legacy. Columbia University, New York

Larson B (2019) IHS introduces recommendations for management of acute dental pain. Indian Health Service, Rockville

Leeds SL (2018) Beyond an emergency declaration: tribal governments and the opioid crisis. U Kan Rev 67:1013

Leston J, Crisp C, Lee C, Rink E (2019) An interview project with Native American people: a community-based study to identify actionable steps to reduce health disparities. Public Health 176:82-91

Long R (2018) Lummi Nation Police Chief

Mack KA, Jones CM, Ballesteros MF (2017) Illicit drug use, illicit drug use disorders, and drug overdose deaths in metropolitan and nonmetropolitan areas-United States. Am J Transplant 17:3241-3252

Meldrum ML (2016) The ongoing opioid prescription epidemic: historical context. Am J Public Health 106:1365

Miller C (2018) Warm springs tribal council representative

Minhee C, Calandrillo S (2019) The cure for America's opioid crisis: end the war on drugs. Harv JL Pub Pol 42:547
Momper SL, Dennis MK, Mueller-Williams AC (2012) Service provider views of OxyContin use on an Indian reservation: traumatic effects on the tribal community. Fam Soc 93:312-318

National Academies of Sciences, Medicine (2017) Pain management and the opioid epidemic: balancing societal and individual benefits and risks of prescription opioid use. National Academies Press, Washington

Occupational Safety and Health Administration (OSHA) (1998) Standard Number 1975.4(b)(3)

Oliphant V (1978) Suquamish Indian Tribe

Park JN, Rouhani S, Beletsky L et al (2020) Situating the continuum of overdose risk in the social determinants of health: a new conceptual framework. Milbank Q 98:700-746

Robbins J (2020) 125 Years Later, Native American Tribe in Montana Gets Federal Recognition. N. Y. Times

Sarpatwari A, Sinha MS, Kesselheim AS (2017) The opioid epidemic: fixing a broken pharmaceutical market. Harv Pol Rev 11:463

Scholl L, Seth P, Kariisa M et al (2019) Drug and opioid-involved overdose deaths-United States, 2013-2017. Morb Mortal Wkly Rep 67:1419

Seven Directions: A Center for Indigenous Public Health (2019) An Environmental Scan of Tribal Opioid Overdose Prevention Responses: Community-Based Strategies and Public Health Data Infrastructure

Smith T (2019) Native Behavioral Health Access Improvement Act of 2019

Stevens S, Andrade R, Korchmaros J, Sharron K (2015) Intergenerational trauma among substance-using Native American, Latina, and White mothers living in the southwestern United States. J Soc Work Pract Addict 15:6-24

Substance Abuse and Mental Health Services Administration (2018) Behavioral Health Services for American Indians and Alaska Natives. Treatment Improvement Protocol (TIP) Series 61. Substance Abuse and Mental Health Services Administration, Rockville

Thumath M, Humphreys D, Barlow J et al (2020) Overdose among mothers: the association between child removal and unintentional drug overdose in a longitudinal cohort of marginalised women in Canada. Int J Drug Policy. https://doi.org/10.1016/j.drugpo. 2020.102977

Tipps RT, Buzzard GT, McDougall JA (2018) The opioid epidemic in Indian country. J Law Med Ethics 46:422-436

U.S. Department of Justice (2017) Indian Country Investigations and Prosecutions

U.S. Health and Human Services (2019) What is the U.S. Opioid Epidemic?

Vashishtha D, Mittal ML, Werb D (2017) The North American opioid epidemic: current challenges and a call for treatment as prevention. Harm Reduct J 14:1-6

Venner KL, Donovan DM, Campbell AN et al (2018) Future directions for medication assisted treatment for opioid use disorder with American Indian/Alaska Natives. Addict Behav 86:111-117

Wendt DC, Marsan S, Parker D et al (2021) Commentary on the impact of the COVID-19 pandemic on opioid use disorder treatment among Indigenous communities in the United States and Canada. J Subst Abuse Treat 121:108165

Zeledon I, West A, Antony V et al (2020) Statewide collaborative partnerships among American Indian and Alaska Native (AI/AN) communities in California to target the opioid epidemic: preliminary results of the Tribal Medication Assisted Treatment (MAT) key informant needs assessment. J Subst Abuse Treat 108:9-19 